\title{
Caracterización de la violencia de pareja en el contexto de la minería aurífera en dos municipios de influencia del páramo de Santurbán, Colombia*
}

\author{
Intimate Partner Violence Characteristics in Two Gold Mining \\ Towns in Santurbán, Colombia between 2008-2015 \\ Caracterização da violência na relação de casal no contexto \\ da mineração aurífera em dois municípios de influência \\ do páramo de Santurbán, Colômbia
}

\author{
René Álvarez Orozco * \\ LUCÍA ANDRADE MANJARRÉS $S^{* * *}$ \\ YinNy PaOla ValenCia AtUesta ${ }^{* \star * *}$
}

FECHA DE RECEPCIÓN: 31 DE ENERO DE 2019. FeCHA DE APROBACIÓN: 5 DE JUNIO de 2019

Doi: http://dx.doi.org/10.12804/revistas.urosario.edu.co/sociojuridicos/a.7634

Para citar: Álvarez Orozco, R., Andrade Manjarrés, L., E Valencia Atuesta, Y. P. (2019). Caracterización de la violencia de pareja en el contexto de la minería aurífera en dos municipios de influencia del páramo de Santurbán, Colombia. Revisa Socio-Jurídicos, 22(1), 175-202. Doi: http://dx.doi.org/10.12804/revistas.urosario.edu.co/sociojuridicos/a.7634

* La presente investigación fue presentada en el VI Congreso Internacional de Ciencias Sociales llevado a cabo en Cancún del 28 al 30 de Noviembre de 2018 en la universidad Tecmilenio en Cancún México y organizado por Global Knowledge Académics. Este artículo es el resultado de una investigación más amplia financiada por la Vicerrectoría de Investigación y Extensión de la Universidad Industrial de Santander, código No. 1969, en el marco de la convocatoria Interna de Investigación para la Consolidación de Centros de Investigación Científica y Tecnológica 20172018 auspiciado por esta entidad y desarrollada dentro de la línea de investigación "Derecho y Estudios de Género" del Grupo de Investigación Derecho y Sociedad GIDES, adscrito a la Escuela de Derecho y Ciencia Política de la Universidad Industrial de Santander.

** Historiador de la Universidad Industrial de Santander y licenciado en Filosofía de la Universidad Santo Tomás de Bogotá. Especialista en Investigación Social de la Universidad Industrial de Santander y magíster en Historia de la misma Universidad. Profesor de Planta adscrito a la Escuela de Derecho y Ciencia Política de la Universidad Industrial de Santander. Coordinador de la Maestría en Derechos Humanos e Investigador del Grupo de Investigación Derecho y Sociedad. Correo electrónico: realvaor@uis.edu.co

*** Abogada de la Universidad Industrial de Santander. Magíster en Igualdad y Derechos Humanos, University of Glasgow. Catedrática de la Escuela de Derecho y Ciencia Política de la Universidad Industrial de Santander. Asesora en asuntos de género en el Consultorio Jurídico de la Universidad Industrial de Santander. Investigadora del Grupo de Investigación Derecho y Sociedad. Correo electrónico: Lucia.Andrade@correo.uis.edu.co

${ }^{\star \star \star \star}$ Abogada de la Universidad Industrial de Santander. Auxiliar de investigación. Investigadora del Grupo de Investigación Derecho y Sociedad. Correo electrónico: Yinny0411@hotmail.com 


\section{RESUMEN}

El presente artículo parte de una investigación que pretende describir y analizar la forma como se configura la violencia de pareja contra la mujer en los municipios de Vetas y California, en el municipio de Santurbán (Santander, Colombia), entre los años 2008 y 2015, desde el análisis estadístico y de discurso. En estos dos municipios se han venido generando una serie de conflictos socio-ambientales que son consecuencia del interés de explotación de los recursos minerales de esta región colombiana. El proyecto desarrollado por la UIS plantea adentrarse en el análisis de los conflictos sociales expresados a través de la violencia de pareja y de género contra la mujer y su relación con la explotación aurífera. Se encontró la existencia de tres factores que subyacen a la violencia de pareja contra la mujer en Vetas y California: una fuerte división sexual del trabajo, la minería y un alto consumo de alcohol. Las autoridades deben crear políticas públicas en estos municipios que den cuenta de la afectación que ha traído para las mujeres de la zona y para sus vidas el el conflicto socioambiental en Santurbán.

Palabras clave: violencia de género, violencia de pareja, Colombia, extractivismo, Santurbán.

\section{ABSTRACT}

This paper seeks to describe and analyze the ways in which intimate partner violence happens in Vetas and California between the years 2008 and 2015. Vetas and California are in the center of a social and environmental conflict due to the gold mining projects in the region (Santurbán). This project uses statistical and discursive analysis to describe and explore the effects that the conflict causes on women, specifically their vulnerability for sufferingintimate partner violence. In that sense, the research shows there are three factors that underline the phenomena in Vetasand California: A strong sexual division of labor, the Mining industry and alcohol abuse. The authorities should create public policies in Vetas and California that address the effects of Santurbán's social and environmental conflict on women.

Keywords: Gender based violence, intimate partner violence, Colombia, mining, Santurbán.

\section{RESUMO}

O presente artigo parte de uma pesquisa que pretende descrever e analisar a forma como se configura a violência de casal contra a mulher nos municípios de Vetas e Califórnia, Colômbia, entre os anos 2008 e 2015 desde a análise estatística e de discurso. Nos municípios de Vetas e Califórnia se tem gerado uma série de conflitos socioambientais resultado do interesse de exploração dos recursos minerais desta região colombiana. O projeto desenvolvido pela UIS propõe se adentrar em análise dos conflitos sociais expressados através da violência de casal e de gênero contra a mulher e sua relação com a exploração aurífera. Se encontrou a existência de três fatores que subjazem à violência de casal contra a mulher em Vetas e Califórnia: uma forte divisão sexual do trabalho, a mineração e um alto consumo de álcool. As autoridades devem criar políticas públicas nestes municípios que deem conta da afetação do conflito socioambiental em Santurbán às mulheres da zona e sua garantia a vivir uma vida livre de violências.

Palavras-chave: violência de gênero, violência de casal, Colômbia, extrativismo, Santurbán. 


\section{Introducción}

Los municipios de Vetas y California, en Santander (Colombia), han estado en el centro del conflicto socioambiental desde 2011, debido a la existencia en el páramo de Santurbán de proyectos de megaminería con empresas como GreyStar, ahora Eco Oro. De acuerdo con Basto (2017), la causa del conflicto fue la pretensión de una multinacional minera canadiense (GreyStar) de explotar a cielo abierto, ${ }^{1}$ lo que afectaría el agua de más de dos millones de habitantes y vulneraría la biodiversidad del complejo. Esto ocasionó la reacción de un grupo de ambientalistas que lograron frenar el proyecto mediante diversas estrategias. En efecto, entre 2012 y 2014 las multinacionales que se encontraban en el páramo entraron en stand by por orden del gobierno, lo que ocasionó un receso en los modos de subsistencia de las comunidades que históricamente han vivido de la explotación de oro, lo que afectó sus economías y estilos de vida. Estos grupos sociales se conformaron en la otra cara del conflicto, ya que los pequeños mineros habían vendido sus títulos a estas compañías, mientras la minería tradicional decaía por las exigencias en el cumplimiento de las normas ambientales (Basto, 2017). En este contexto, no se ha tenido en cuenta el impacto del conflicto socioambiental en la vida de las mujeres y en su derecho a vivir una vida libre de violencias. Esta investigación provino precisamente de la necesidad de explorar esa realidad invisibilizada y tuvo como objetivo describir y analizar cómo se configura la violencia de pareja contra la mujer en los municipios de Vetas y California entre los años 2008 y 2015, por medio de un análisis estadístico y de discurso.

La metodología utilizada fue mixta, de carácter descriptivo y exploratorio; además, se utilizaron técnicas como el análisis documental y

Eco Oro Minerals Corp, para ese entonces GreyStar, solicitó licencia ambiental para que se fuera concedida la exploración acuífera a gran escala y a cielo abierto en el Páramo de Santurbán, bajo el proyecto llamado Angostura, el cual consistía en la intervención de más de 1.104 hectáreas de páramo (Zárate, 2011). Luego de multitudinarias marchas e incidencia social y política, se expidió la resolución 2090 de 2011 del Ministerio de Ambiente, que delimita la zona de páramo y la zona de explotación minera, en el marco de la ley 1450 de 2011, que prohíbe todo tipo de explotación minera en zona de páramo. En 2013 la CDMB, a través del acuerdo 1236 de 2013, declara la zona como parque natural regional. En 2017 la sentencia T-631 de 2017 ordena la redelimitación del páramo debido a fallas en el proceso de delimitación de 2011 y pide que se tome en cuenta la realidad económica y social de los municipios afectados Vetas y California. 
estadístico, así como entrevistas a funcionarios encargados de la protección de las víctimas de violencia de pareja en el municipio.

A partir de este ejercicio se encontró la intersección de tres factores: una fuerte división sexual del trabajo, la minería y el consumo de alcohol como elementos distintivos que subyacen a las dinámicas de violencia en la relación de pareja en los municipios estudiados.

Lo anterior pone sobre la mesa la neesidad de generar una perspectiva de política pública que analice las consecuencias que ha traído para las mujeres vetanas y californianas el conflicto socioambiental del páramo de Santurbán; dicha perspectiva debe entender cómo las dinámicas económicas y socioculturales relacionadas con la minería y sus altibajos afectan el derecho de las mujeres a una vida libre de violencias y el cumplimiento de la ley 1257 de 2008.

\section{Marco contextual y teórico}

\section{Violencia de género}

La cultura patriarcal construye sociedades profundamente divididas por unas relaciones de poder mediadas por el sexo y el género de las personas. Dichas brechas de género, mediadas por la clase, la raza y la religión, entre otros, dan lugar a la creación de estereotipos de género.

Para Cook y Cusack, los estereotipos de género se refieren a "la construcción social y cultural de hombres y mujeres debido a sus diferentes funciones físicas, biológicas, sexuales y sociales. Más ampliamente pueden pensarse como las "convenciones que sostienen la práctica social del género" (2011, p. 23).

Estamos frente a una generalización en la cual, por el hecho de pertenecer a un grupo en particular de hombres o mujeres, se asignan unas características comunes mediadas por la asignación de sexo y género de la persona.

Además, para estos autores las tensiones empiezan cuando se difumina la individualidad de los seres humanos y se niegan sus deseos y necesidades individuales, creándose jerarquías de género (2011). 
Lo anterior implica que los estereotipos de género en una cultura patriarcal dan lugar a generalizaciones sobre los seres humanos que niegan su capacidad individual de desarrollar su proyecto de vida; además, las jerarquías de género son un factor de control y discriminación sobre aquellos y aquellas que no entran en dichos cánones sociales.

En estos términos lo explica Facio y Fries al referirse a la ideología sexual

Una ideología sexual toma a uno de los sexos como parámetro de lo humano, además explica las relaciones entre hombres y mujeres, sus deberes y responsabilidades (estereotipos sobre los roles sexuales), el sistema justifica las reacciones negativas frente a quienes no se conforman, asegurando así el mantenimiento del estatus quo. (Facio \& Fries, 2005, p. 261)

Frente a los estereotipos de género y sus jerarquías, Cook y Cusack advierten de su resilencia, dominancia y persistencia, condiciones sine qua non para que exista la estratificación y subordinación social de las mujeres (2011).

Bourdieu (2000) hace énfasis en este escenario a partir del estudio de las formas de dominación que persisten en el inconsciente individual y colectivo. Para él, la mujer aparece como un ser percibido, es decir, que se desarrolla únicamente a través de las necesidades del hombre y depende de este para hallar su identidad. Dentro de su familia, el hombre es quien determina las vocaciones o roles estereotipados que cumplirán los individuos, los cuales serán naturalizados con el tiempo y perpetuados en la sociedad. Este ejercicio se ha denominado violencia simbólica de la cual particularmente el padre, tiene su monopolio (Bourdieu, 2000). Es así como el padre ordena, la madre refuerza la norma y los hijos apropian y reproducen de forma inconsciente los roles sociales asignados.

Este terreno de lo simbólico ancla en el inconsciente la asignación jerarquizada de roles entre hombres y mujeres, asegurando su reproducción social; ello nos lleva a decir que la generación de estereotipos discriminatorios y de violencias contra quienes se salen de dichos roles tiene también asidero en lo simbólico. 
Para dar ejemplo de ello, Bourdieu (2000) hace referencia a la violencia sufrida por las primeras mujeres que entraron a asumir puestos laborales socialmente representados desde lo masculino, la cual no fue sino una reacción de defensa de los hombres frente a lo que tradicional y culturalmente se les había asignado, y a su propia identidad masculina.

Teniendo en cuenta lo anterior, Galtung analiza la generación de la violencia, encontrando que existen tres elementos que aseguran su perpetuación social: la violencia simbólica, estructural y directa.

El autor concuerda con Bourdieu en la noción de violencia simbólica al considerarla como un medio de legitimación y asimilación de violencias directas y estructurales, es decir, es la fuente de reproducción social de estereotipos y relaciones de dominación y violencia (Galtung, 1989).

Dicha violencia simbólica o cultural permite que violencias más visibles aparezcan, esta es una constante mientras que la violencia estructural hace referencia a procesos de explotación, marginación y fragmentación del tejido social; por tanto, la violencia directa es un acontecimiento claramente medible y cuantificable como la muerte, el acoso, las mutilaciones entre otros, mientras la relación entre símbolos, estructura y acontecimientos violentos es interdependiente (Galtung, 1989).

Desde una perspectiva de género, dicha tipología es fundamental para entender cómo el patriarcado retroalimenta constantemente relaciones violentas de dominación hacia las mujeres. La violencia simbólica está presente en el arte, las enseñanzas en las escuelas, la iglesia y la familia, en las cuales se legitima la subordinación de la mujer al considerarla como natural. A su vez, la violencia estructural hace relación a los factores que impiden la movilidad social de las mujeres, por ejemplo, la recarga en las labores de cuidado, la feminización de la pobreza, el bajo acceso a anticonceptivos y a servicios de salud sexual y reproductiva, en general; lo anterior confluye en situaciones de violencia directa como los feminicidios, la violencia sexual y la violencia de pareja, entre otras.

Se hace necesario, entonces, trasladar el análisis de la violencia de género al ámbito de las relaciones íntimas de las mujeres y la violencia de pareja habida cuenta de que una gran proporción de las violencias de género se dan en el marco de la familia y de las relaciones de noviazgo además de que esto escenarios son propicios para la reproducción de un orden social patriarcal. Si bien se entiende que no es el único escenario 
en el que se da la violencia de género y la misma no se presenta únicamente contra las mujeres.

Para autores como Facio, Fries y Bourdieu, entre otros de la corriente psicoanalítica del feminismo, la familia es un lugar donde desde temprana edad se reproduce la violencia; en este sentido, Facio y Fries lo expresan de la siguiente forma:

La familia es un lugar privilegiado para la reproducción del patriarcado en tanto constituye la unidad de control económico, sexual y reproductivo del varón sobre la mujer. La familia ha significado una forma de enriquecimiento del varón sobre la mujer y los hijos. La invisibilización del aporte de la mujer a la reproducción social de la comunidad. (Facio G Fries, 2005, pp. 285-286)

En conclusión, dentro de los hogares y en el marco de las relaciones afectivas se producen violencias de género que buscan el control social de la mujer y reafirman un estatu quo hegemónico de superioridad masculina. La existencia previa a la relación afectiva de un contexto patriarcal aumenta la posibilidad de violencias de género contra la mujer en las relaciones romántico-afectivas. De esta forma lo resume Maqueda Abreu:

(las relaciones afectivas) Son situaciones de riesgo no ya sólo por la naturaleza y complejidad de la relación afectiva y sexual, por su intensidad y por su privacidad sino, sobre todo, porque constituyen un espacio privilegiado para el desarrollo de los roles de género más ancestrales, esos que reservan a la mujer los clásicos valores de subjetividad, cuidado y subordinación a la autoridad masculina. La posición hegemónica del varón garantiza la continuidad de esas expectativas, en la familia o fuera de ella, y se hace valer a toda costa, a menudo con el recurso a la violencia. (Maqueda, 2006, p. 4)

Uno de los modelos que permiten entender las dinámicas de la violencia de pareja contra la mujer desde una perspectiva de género es el modelo ecológico. Dicha teoría se basa en la intersección del ámbito individual o personal, el microsistema o aspectos de la dinámica cotidiana de la pareja y el exosistema o las instituciones que rodean a la pareja y 
que desde afuera se convierten en factores protectores o por el contrario hacen perdurar el maltrato (Alcenar-Rodríguez \& Cantera, 2012).

Este punto de vista de la violencia de pareja permite entender cómo la cultura patriarcal permea las relaciones afectivas mediante la historia personal de los individuos y los discursos de instituciones como la familia, la iglesia y el Estado, entre otros. Allí puede insertarse como factores estresores que da lugar a la violencia en la relación de pareja, la inequidad económica en la pareja, la pobreza, la socialización de roles de género violentos desde la infancia y las sociedades cerradas fijadas con una fuerte subordinación.

En conclusión, las relaciones de pareja y familiares son un escenario de reproducción de la cultura patriarcal y de roles de género sexistas y violentos. En este escenario, la violencia se da como una forma de mantener el control y de preservar un estatu quo de dominación masculina; existen elementos que favorecen y perpetúan la violencia de género en la relación de pareja desde la experiencia personal de los individuos, la cotidianidad de la pareja y las instituciones que la rodean.

\section{Minería y configuraciones de género}

En Colombia, las denuncias por vulneración de los Derechos Humanos y por la intensificación del conflicto armado en un contexto de explotación minera son constantes; por si fuera poco, la riqueza mineral de las zonas ha llevado a que se asienten en ellas grupos armados al margen de la ley, que buscan el control territorial de las minas. Ejemplo de ello es lo que ocurre con la explotación de oro en el Pacífico colombiano, la explotación de carbón en el Cesar, de petróleo en el Magdalena Medio, de petróleo y oro en la Amazonía colombiana y de esmeraldas en Boyacá, entre otros. Producto del conflicto que se crea entre los grupos armados ilegales y la sociedad civil, Colombia se debate en los primeros lugares de asesinato de líderes ambientales en el mundo (El Espectador, 2018). Los informes de CENSAT AGUAVIVA (Bermúdez, 2012), del CINEP, y del Instituto Nacional de Memoria Histórica INMH han reportado esta situación exhaustivamente.

El contexto anterior afecta de forma especial a mujeres y niñas, quienes son víctimas desproporcionadas de violencia sexual (BBC, 2016) y 
desplazamiento forzado, además de situaciones de pobreza y marginalidad generalizadas.

Bermúdez (2012) resume la situación así:

En los entornos mineros se ha acentuado la demanda de servicios sexuales, principalmente, por parte de empleados y obreros de la industria minera. Adicionalmente, aparecen situaciones críticas que afectan directamente a las mujeres, tales como la servidumbre, la trata de personas, la migración de mujeres para prestar servicios sexuales (los llamados servicios "prepago") y la estigmatización de aquellas que ejercen la prostitución. (p. 9)

La intensificación de la violencia de género en zonas de extracción minera se da en un contexto en el cual existen unas relaciones de género basadas en la división sexual del trabajo y la marginalidad económica.

Guiza (2013) y Chaparro (2005), en sus estudios sobre la explotación de oro en Colombia y Bolivia, muestran cómo las mujeres son relegadas a labores de cuidado como la preparación de alimentos, pero no ejercen como mineras, ya que no están capacitadas para ello. Así, "durante todo el proceso de extracción de oro las actividades son realizadas por hombres porque requiere de condiciones especiales, ellos cumplen con todos los requisitos" (Chaparro, 2005).

La Red Nacional de Mujeres en 2015 refuerza este argumento citando a Miriam Gartor (2014), cuando afirma que:

Este proceso (la minería en Colombia) instala en las comunidades una economía productiva altamente masculinizada que acentúa la división sexual del trabajo. El resto de economías no hegemónicas, la economía popular, del cuidado, etc., que hasta el momento de la intervención han tenido cierto peso en las relaciones comunitarias, pasan a ser marginales. (Red Nacional de Mujeres, 2015, p. 2)

Como zona minera, Santurbán no escapa de esta realidad; una de las entrevistadas menciona que las mujeres suelen asumir tareas relacionadas con el cuidado, la preparación de alimentos para las empresas mineras y la seguridad ocupacional (Participante 2, Comunicación personal, 3 de noviembre de 2017). Esto también queda en evidencia ya que en más 
del 50\% de los casos estudiados de violencia de pareja en esta investigación, la víctima es ama de casa. En este sentido, estudios realizados en Chile dan cuenta de que dicha división sexual del trabajo acentuada por las labores mineras es perpetuada y construida dentro de la familia.

La permanencia de esta división sexual y social entre hombres y mujeres se encuentra ligada a los requerimientos de las actividades laborales propias de la minería, desde los cuales se mantiene el rol de proveedor y alta sexualidad asociadas con "lo masculino" a la vez que se justifica la omisión de actividades de cuidado y crianza de hijos para los hombres. Mientras que se perpetúan roles de cuidado de infantes, relegación al espacio privado y control de las experiencias sexuales en las mujeres. Esto da cuenta de cómo la dimensión económica y laboral propia de la región se convierten en un eje estructurante de las funciones familiares. Para la mantención de este orden productivo se han desplegado tecnologías de gobierno mediante las cuales se realiza categorización social y jerarquización de las mujeres de acuerdo a su adscripción y cumplimiento de estas normatividades de género y sexualidad. (Contreras, 2014, p. 451)

En el marco de la minería, este control es germen de violencia y discriminación contra las mujeres. En el caso del suroccidente de Boyacá, Colombia, existe una relación fuerte entre la división sexual del trabajo relacionada con la minería y la generación de procesos de violencia y discriminación contra las mujeres y niñas en sus relaciones cotidianas e intrafamiliares, por parte de padres, hermanos y compañeros (Parra, 2006).

En el contexto de Boyacá, la transición de la agricultura a la minería llevó a una intensificación de conflictos en el hogar relacionados con la posesión de armas de fuego y el consumo de alcohol; además, los roles de género cambiaron en tanto que el hombre se ausenta del hogar para trabajar en la mina mientras la mujer se queda ejerciendo labores de cuidado del hogar y de las fincas. Luego, si bien la mujer empieza a ejercer labores de minería, la violencia y discriminación no cesaron (Parra, 2006).

La investigadora y profesora de la Universidad Industrial de Santander, Raquel Méndez Villamizar, en su artículo "Tensiones identitarias en las vivencias erótico-afectivas: escenario cotidiano de las relaciones de género en contextos extractivos de oro y petróleo" demuestra la existencia 
de relaciones de poder, que impiden a las mujeres beneficiarse de los ingresos de la minería y mejorar sus condiciones de vida en relación con los hombres. La autora pone en evidencia que, a pesar del auge de la minería en las veredas Angosturas y La Baja, en California (Santander, Colombia), la cultura patriarcal no se pone en crisis ni se transforman las relaciones entre hombres y mujeres.

No se evidencia una incidencia directa en el incremento de las libertades, el reconocimiento de la dignidad y el acceso a las oportunidades requeridos para ampliar la "potencia de sí» de las mujeres en los contextos extractivos de oro y petróleo en los dos casos de estudio. El mundo intersubjetivo de la vida cotidiana rural con su referencial significativo (Schutz, 2003) tensiona a favor del mantenimiento de las desigualdades sexuales en menoscabo del ejercicio de los derechos humanos de las mujeres". (Méndez, Rojas \& Montero, 2016, p. 350)

Partiendo del contexto anterior, es importante mirar si los procesos de dominación masculina de Boyacá y las tensiones que presenta Méndez, Rojas y Montero en California, Santander se replican a la hora de hablar de violencia en la relación de pareja en los municipios de Vetas y California, en el páramo de Santurbán. En este sentido, se realizó un diagnóstico de las dinámicas de violencia de pareja partiendo de una caracterización socioeconómica de la violencia, para luego hacer una exploración de los discursos socio jurídicos que la rodean.

\section{Metodología}

Para la construcción del panorama criminológico de la violencia de pareja contra la mujer, se desarrolló una investigación socio-jurídica de carácter descriptivo. La investigación busca analizar la realidad social y actúa sobre los comportamientos sociales regulándolos para que sea posible alcanzar los fines del Estado. En este caso, la prevención, a partir de políticas públicas, de la violencia de género contra la mujer (Giraldo Ángel, 2012).

Entonces, este documento parte de una investigación que tuvo como objetivo describir y analizar cómo se configura la violencia de pareja 
contra la mujer en los municipios de Vetas y California desde el análisis estadístico y de discurso en el periodo comprendido entre 2008 y 2015. El análisis de discurso desarrollado fue de tipo crítico con las características establecidas por Fairclough y Wodak (1997); dicho análisis abordó las relaciones de poder existentes en el discurso de los funcionarios públicos y si las mismas representaban una barrera en el acceso efectivo a la justicia para las mujeres víctimas de violencia en la relación de pareja. En este artículo se hace referencia a los primeros resultados de la investigación alrededor de la caracterización socioeconómica de la violencia en la relación de pareja en Vetas y California, Santander.

El método utilizado fue mixto, ya que incluyó un análisis cuantitativo y cualitativo de la información. Se escogió específicamente el tema de violencia de pareja contra la mujer dentro de las diversas violencias de género que existen por la facilidad de acceso a dicha información y porque la cantidad de casos es significativamente mayor a otros tipos de violencia. El fin último de esta investigación fue sentar una base en materia de políticas públicas efectivas en estos municipios que prevenga y trate adecuadamente la violencia de pareja contra la mujer.

Esta investigación se enfocó en los municipios de Vetas y California, debido a que han estado en el centro de un conflicto socioambiental de grandes proporciones, del cual poco se ha analizado su impacto social, como se expresó en la introducción. La investigación se desarrolló en dos fases: en la primera se caracterizó la violencia en la relación de pareja, de acuerdo con unas variables estadísticas definidas; y luego, un análisis sobre el acceso a la justicia de las víctimas, a partir de entrevistas a funcionarios judiciales y administrativos encargados de la protección y atención de esta población. Para la realización de este artículo se muestran los resultados estadísticos de la primera fase de la investigación, cuya metodología constó de los siguientes pasos.

En primer lugar, se realizó un análisis de las violencias de género contra la mujer presentes en contextos de extracción minera. Segundo, se hizo una exploración de fuentes primarias sobre violencia de pareja contra la mujer en las comisarías de familia y en las casas de justicia, juzgados, la Fiscalía seccional de Bucaramanga y el Instituto Nacional de Medicina Legal y Ciencia Forense (INML). Esta exploración incluyó la revisión documental de las denuncias interpuestas por violencia intrafamiliar y 
de género. Tercero, se caracterizaron los datos estadísticos obtenidos de acuerdo con las siguientes variables: la edad, la ocupación, el número de hijos, el grado de escolaridad, los antecedentes penales, el lugar, la forma de la agresión, el contexto en el que se cometió la agresión, la zona/comunas, el tipo de relación de la víctima y del victimario, y el motivo aducido. Las variables son obtenidas por medio de la exploración conceptual del tema y algunas surgieron de la revisión de expedientes. Cuarto, se entrevistó a las autoridades encargadas de la atención de las mujeres víctimas de violencias de pareja en los municipios para enriquecer el análisis estadístico; por último, se analizaron los datos obtenidos desde una perspectiva de género y con especial énfasis en el efecto de la minería sobre la violencia de pareja contra la mujer.

Además, se debe tener en cuenta que no existen ni en los municipios ni en el país estadísticas unificadas frente a la violencia de género, por lo que no hay un panorama claro sobre este flagelo. En este estudio encontramos que para el municipio de California dos entidades tienen cifras por el delito de "violencia intrafamiliar": la Fiscalía General de la Nación y el Instituto Nacional de Medicina Legal. Por otra parte, se tiene que solo el INML tiene las cifras disgregadas de acuerdo con la relación agresor-víctima, es decir, solo en estas cifras se puede determinar el alcance de la violencia de pareja contra la mujer en Vetas y California.

Sin embargo, el INML refirió en múltiples oportunidades que el alcance que tiene en los municipios estudiados es menor, ya que depende de que el médico adscrito al servicio social obligatorio realice el reporte de la información pericial para el dictamen medico legal; además, durante 2010 no hubo médico que realizara dichos procesos en California. ${ }^{2}$

En cuanto a las cifras de la Fiscalía, estas no muestran la heterogeneidad del delito de violencia intrafamiliar, ya que no establece la relación entre la víctima y el agresor. Por tanto, solo se tienen cifras generales que dan cuenta de 14 casos por violencia intrafamiliar en el municipio de California ocurridos entre 2006 y 2016. En el caso de Vetas, existen 13

2 Oficio No 026-GCRRV-DRNORIENTE-2017 y oficio 073-GCRRV-DRNORIENTE-2017 de fecha de 23 de marzo y 8 de agosto de 2017, expedidos por Medicina Legal en respuesta a sendos derechos de petición enviados en el marco de la investigación. 
casos de violencia intrafamiliar de conocimiento de la Fiscalía General de la Nación entre los años 2007 y 2016.

Por todo lo anterior, se acudió a las comisarías de familia de los municipios, quienes permitieron el acceso a los expedientes de los procesos por violencia intrafamiliar para realizar una aproximación más acertada al fenómeno de la violencia de pareja. Por otra parte, se requiere de fondo complementar el análisis ofrecido con grupos focales en la comunidad, que permitan contrastar las conclusiones de este proceso y llegar a resultados que sustenten políticas públicas efectivas para tratar la violencia de pareja contra la mujer.

\section{Resultados}

Estos resultados fueron producto de un proceso de documentación y análisis estadístico combinado con entrevistas a autoridades de los municipios de Vetas y California. Cabe aclarar que si bien la investigación hace referencia a los años comprendidos entre 2008 y 2015, la realidad encontrada en campo es que en el municipio de California no fue posible acceder a los registros anteriores a 2010 porque no estaban organizados ni diferenciados de otros procesos judiciales y administrativos. Con dicha salvedad se procede a presentar los resultados de la primera fase de la investigación.

Esta investigación se pregunta sobre las consecuencias que ha traído para la vida de las mujeres de la zona la delimitación inicial del páramo de Santurbán en 2011 y el conflicto socioambiental generado, lo anterior teniendo en cuenta que, al analizar las fechas de denuncia de violencia en la relación de pareja en los municipios de Vetas y California, desde 2011 la cifra ha aumentado hasta alcanzar su pico en 2013,3 para luego disminuir. ${ }^{4}$ Entonces, Los conflictos socioambientales deben ser un factor

3 En California, el $33 \%$ de los casos estudiados fueron denunciados en 2013; en Vetas esta proporción fue el $22 \%$, según datos recogidos en las comisarías de familia.

4 Estos datos se obtuvieron a través de las indagaciones realizadas en las comisarías de familia. 
central en la prevención y atención de las violencias de género en las relaciones de pareja en Santurbán.

Por otro lado, en las dinámicas de la violencia de pareja contra la mujer fue posible encontrar elementos comunes con la realidad regional y nacional. Gran parte de las mujeres víctimas de violencia de género en la relación de pareja en Vetas y California están entre los 20 y los 35 años de edad, ${ }^{5}$ las violencias más denunciadas son la psicológica y física ${ }^{6}$ y el hogar es el lugar más inseguro para las mujeres. ${ }^{7}$

Ello tiene relación con lo que expone el Informe Forensis Masatugó del Instituto Nacional de Medicina Legal y Ciencia Forense (INMLCF), que se realizó entre 2009 y 2014. En él quedó en evidencia que el 70,81 \% de las violencias de pareja contra la mujer conocidas por el INMLCF ocurrió en la vivienda de la víctima (INMLCF, 2015, p. 144). Las mujeres que están entre los 18 y los 39 años ocupan el mayor porcentaje de las violencias de pareja conocidas por el INMLCF, con 231.081 casos (INMLCF, 2015, p. 139).

Al mismo tiempo se encontró que existen tres factores relacionados con la violencia en la relación de pareja y que están conectados con la realidad social y minera de Vetas y California: estos son la minería, el alcohol y una fuerte división sexual del trabajo.

La exposición de resultados finalizará con un aparte acerca de las validaciones de la violencia de pareja en los municipios estudiados.

\section{La división sexual del trabajo y la dependencia económica de la mujer}

Para entender la división sexual del trabajo como factor que subyace a la violencia de género se partirá de una concepción dicotómica de género resumida de forma acertada por Alda Facio y Lorena Fríes; según las autoras:

5 Según las comisarías de familia, el $40 \%$ de las mujeres que denunciaron violencia de pareja en Vetas tenía entre 25 y 34 años. Este porcentaje asciende al $75 \%$ en el caso de las mujeres entre 20 y 34 años en California.

6 Las violencias más denunciadas fueron la psicológica y la física, el $77 \%$ de los casos en Vetas y el $66 \%$ en California.

7 El $85 \%$ de las violencias de pareja denunciadas en el municipio de Vetas se dieron en el hogar. En California, el porcentaje fue del $83 \%$. 
El concepto de género alude tanto al conjunto de características y comportamiento como a los roles, funciones y valoraciones impuestas dicotómicamente a cada sexo a través de procesos de socialización, mantenidos y reforzados por ideologías e instituciones patriarcales. (Facio E Fries, 2005, p. 271)

La economía no está por fuera de los procesos de socialización de género y es por tanto una Institución patriarcal. A las mujeres les está reservado el trabajo reproductivo y de cuidado, mientras que los hombres tienen a su cargo el trabajo productivo (Federici, 2013).

En California y Vetas existe una profunda división sexual del trabajo en las mujeres víctimas de violencia en la relación de pareja y sus agresores. La ocupación de la víctima más referenciada es la de ama de casa tanto en Vetas como en California. Lo anterior se complejiza si, como se ve en este acápite, las mujeres agredidas tienen baja escolaridad, suelen ser menores de 40 años y con hijos menores de edad a su cargo. Este perfil acentúa la dependencia económica de la mujer, lo que, de acuerdo con Amartya Sen (citada por Pineda \& Otero, 2004) y a CEPAL, afecta la capacidad de la mujer de terminar con ciclos de violencia de pareja.

Figura 1. Ocupación de la víctima en Vetas. Datos comisaría de familia*

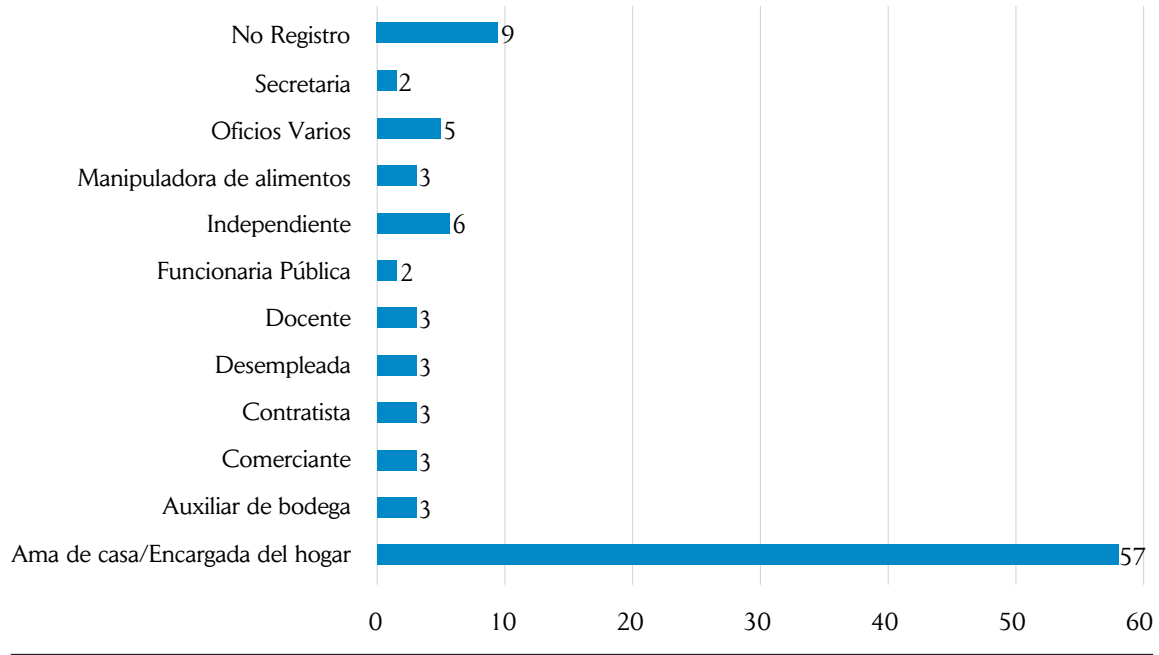

${ }^{*}$ Datos calculados en porcentajes.

Fuente: elaboración propia. 


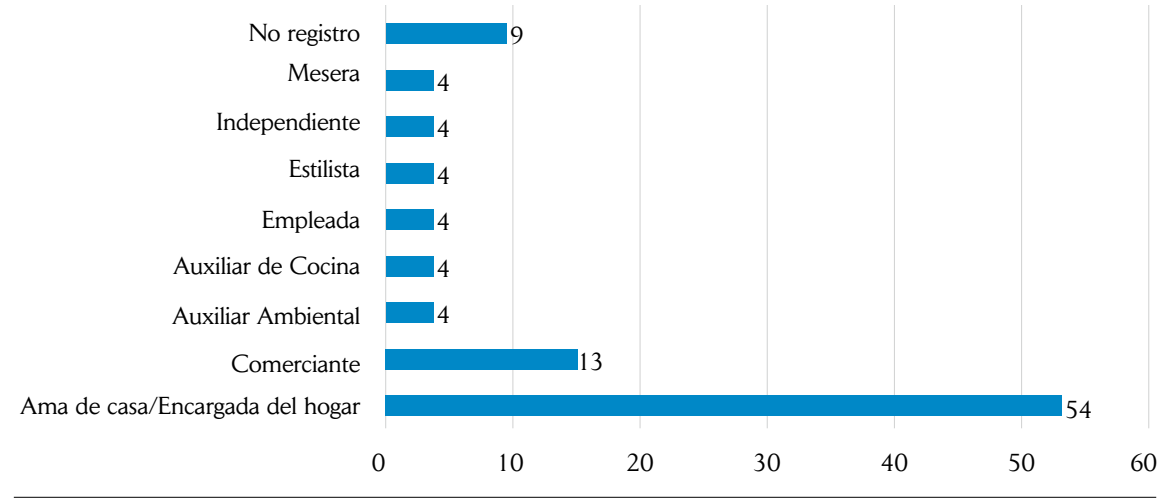

*Datos calculados en porcentajes.

Fuente: elaboración propia.

Las mujeres empleadas, lo son en oficios considerados femeninos como estilista, secretaria y auxiliar de cocina. A su vez, como se expresa anteriormente, las mujeres que hacen parte de la industria minera lo son en cargos asistenciales, como auxiliar ambiental, auxiliar de bodega y cocinera. Esto fue corroborado por el participante P2, quien dijo que las empresas promueven el empleo de las mujeres en áreas consideradas femeninas (Participante P2, comunicación personal, 3 de noviembre de 2017).

De acuerdo con investigaciones realizadas por Chaparro (2005), Bermúdez (2012), Contreras (2014) y la Red Nacional de Mujeres (2015), en Bolivia, Colombia y Chile existe una división sexual del trabajo en la cual la mujer queda fuera de la minería, centrándose en labores menos productivas y convirtiendo al hombre en el proveedor del hogar.

En cuanto a los hombres, si bien la ocupación no es una variable que se referencie de forma habitual por parte de las comisarías de familia, en los casos donde se ha registrado la ocupación del agresor pululan las labores típicamente masculinas, como ser guardia de seguridad, conductor y minero. En cuanto a la variable "independiente", una de las autoridades entrevistadas mencionó que, por lo general, los casos de violencia que suceden en el municipio hacen referencia a las personas que ejercen la minería por fuera de empresas formalmente constituidas. ${ }^{8}$ (Participante 3 , comunicación Personal, 19 de junio de 2018).

8 Participante P3. 
Figura 3. Ocupación del agresor en Vetas. Datos comisaría de familia*

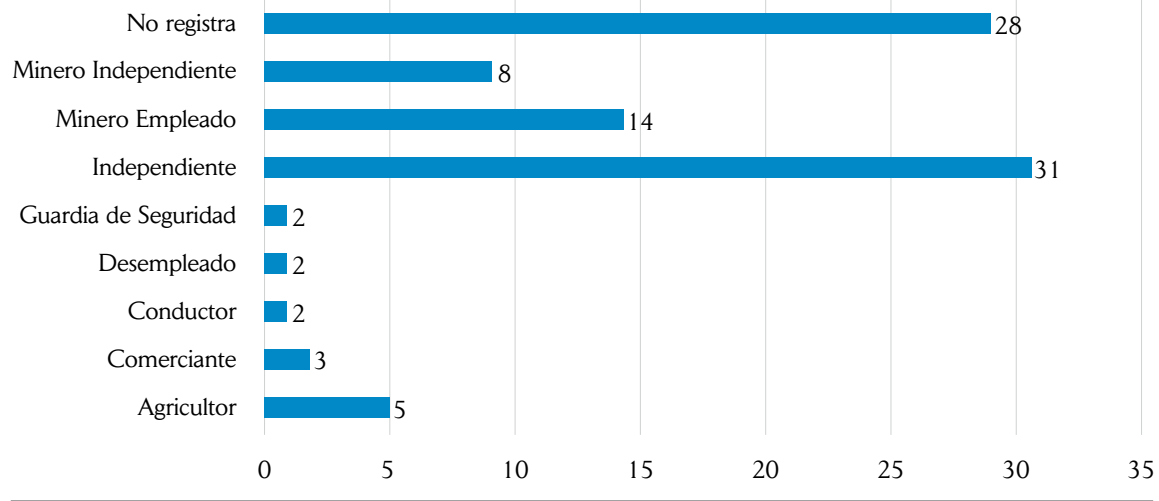

${ }^{*}$ Datos calculados en porcentajes.

Fuente: elaboración propia.

Figura 4. Ocupación de la vítima en California. Datos comisaría de familia*

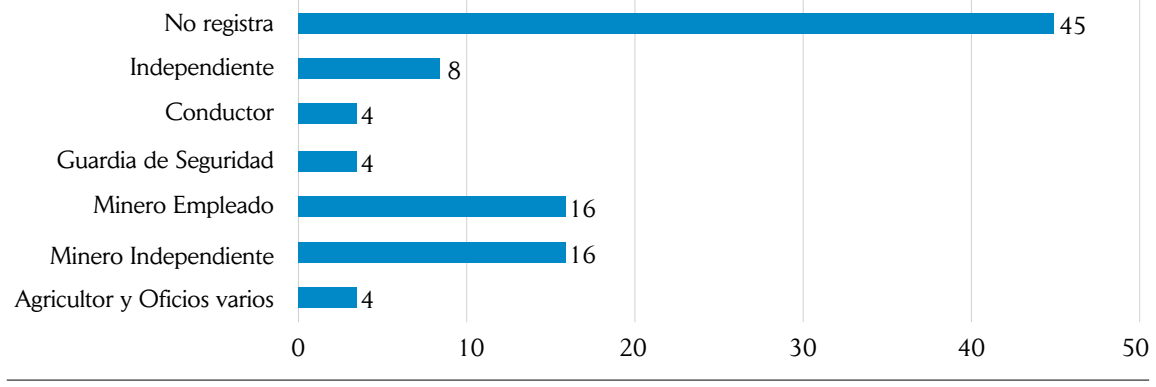

*Datos calculados en porcentajes.

Fuente: elaboración propia.

La situación de dependencia económica de las mujeres en los municipios estudiados se encuentra ejemplificada por la Red Nacional de Mujeres (2015), cuando ellas denuncian la marginalidad económica de las mujeres mineras en Colombia.

Lo anterior debe entenderse en un contexto donde las tasas de desempleo son bastante altas. En los últimos años, el desempleo en Vetas ha escalado al 70 y $80 \%$ entre la población apta para trabajar (Vanguardia Liberal, 2018). En su plan de desarrollo 2012-2015, la alcaldía de Vetas expresó con cifras de la RED UNIDOS que la tasa de desempleo rondaba el 20\% para 2012 (Alcaldía de Vetas, 2012); según el alcalde, la escalada del desempleo se debe a la crisis del sector de la minería en la zona. 
En este contexto, aplicar las teorías de la negociación de Amartya Sen desde un enfoque de género permite entender cómo operan las relaciones de poder en el marco de las relaciones afectivas. Pineda y Otero hacen un análisis de la violencia doméstica y de pareja desde la negociación y renegociación de los intereses de las partes involucradas.

Sen explica cómo en las relaciones afectivas y familiares aparecen elementos de cooperación y conflicto donde la pareja coopera para obtener unos intereses comunes $\mathrm{y}$, al mismo tiempo, tiene que negociar sus intereses individuales, en muchos casos contrapuestos. Quienes tienen mayor poder de negociación son aquellos que, en caso de que la relación fracase, podrían tener un mínimo de calidad de vida y sus relaciones sociales no se verían tan afectadas (Pineda \& Otero, 2004).

Desde una perspectiva de género es posible entender cómo las representaciones y roles de género se articulan con este modelo de negociación, ya que quienes socialmente están siendo considerados en roles subordinados, como las mujeres, tienen históricamente menor poder de negociación y mayores posibilidades, por ejemplo, de dependencia económica (Pineda \& Otero, 2004).

Esta dependencia económica de las mujeres víctimas, en el caso de California y Vetas, se agrava debido a que las mujeres víctimas analizadas en el marco de la presente investigación tienen una educación básica y media ( $42 \%$ de las mujeres víctimas de violencia estudiadas en Vetas y $20 \%$ de las mujeres en California) (Comisaría de Familia de Vetas y California, 2018) y pocas ascienden a niveles técnicos o profesionales (en Vetas el 14\% de las mujeres víctimas son estudiadas, mientras que en California ese porcentaje es del 4) (Comisaría de Familia de Vetas y California, 2018). Sin embargo, a pesar de que los hombres tienen menor nivel educativo (En Vetas el $14 \%$ de los hombres agresores han culminado la secundaria, mas no han alcanzado niveles ni técnicos ni profesional, mientras el $4 \%$ en California han culminado la secundaria) (Comisaría de Familia de Vetas y California, 2018), sus posibilidades de emplearse y acceder a una remuneración son mayores (como se puede ver en las gráficas 1 a la 4); esto nos permite afirmar que estudiar por sí solo no implica para la mujer mayores posibilidades de autonomía económica que le permita blindarse ante situaciones violentas. 
Un resultado importante es que el $100 \%$ de las mujeres víctimas en ambos municipios tienen por lo menos un hijo con el agresor (Comisaría de Familia de Vetas y California, 2018). Esto ahonda la brecha económica que sufren las mujeres, ya que están obligadas a mantener una relación permanente con quien las agrede en pos de la estabilidad económica de sus hijos, sometiéndose no solo ellas a la violencia intrafamiliar, sino también a los niños que tienen a cargo.

A pesar de estos importantes hallazgos, las variables ocupación, número de hijos y nivel educativo no aparecen prima facie en los documentos de denuncia de las violencias de pareja, así como no existe un análisis serio sobre la dependencia económica de las mujeres; en este orden de ideas, los operadores de justicia (comisarios y jueces) invisibilizan la violencia económica, entendida en la Ley 1257 de 2008, Art 2 como "cualquier acción u omisión orientada al abuso económico, el control abusivo de las finanzas, recompensas o castigos monetarios a las mujeres por razón de su condición social, económica o política".

Sin emabargo, la violencia económica y patrimonial no es entendida como violencia ni por los funcionarios y funcionarias, ni por las víctimas (Comisaría de Familia de Vetas y California, 2018); aun cuando es posible observar situaciones de dependencia económica, ya que, como se anotó con antelación, la mayoría de las víctimas cuyos casos llegaron a la Comisaría de ambos municipios eran amas de casa. A su vez, muchas mujeres, si bien no mencionaron la violencia económica, sí pidieron al finalizar la audiencia por violencia intrafamiliar que "le pase alimentos a la niña" o comentarios como "le pido, por favor, que me ayude con un mercadito" (Comisaría de Familia de Vetas y California, 2018).

\section{La minería y el consumo de alcohol, contextos} que favorecen las violencias en la relación de pareja

Todos los participantes hicieron referencia al alto consumo de alcohol en el municipio (comunicación personal, 2018). Lo anterior se agrava con el hecho de que un alto porcentaje de casos de violencia en los municipios estudiados se dieron bajo la influencia del alcohol y otras sustancias psicoactivas en el agresor (94\% de los casos en Vetas y 58\% de los casos en California) (Comisaría de Familia de Vetas y California, 
2018). En algunos casos, las situaciones de violencia se dieron en bares, o saliendo de estos lugares de diversión. ${ }^{9}$ Esto se relaciona con lo que Parra (2006) describe en el suroccidente de Boyacá.

Figura 5. Consumo de alcohol en Vetas. Datos comisaría de familia*

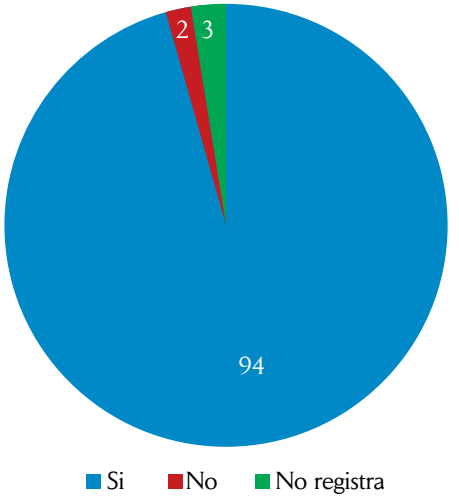

*Datos calculados en porcentajes

Fuente: elaboración propia.

Figura 6. Ocupación de alcohol por parte del agresor en California.

Datos comisaría de familia*

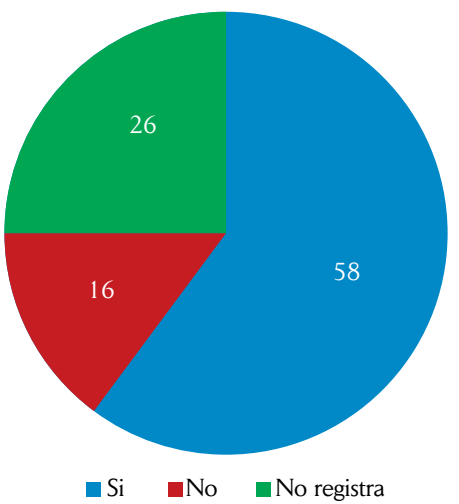

${ }^{*}$ Datos calculados en porcentaje.

Fuente: elaboración propia.

9 Expedientes de violencia intrafamiliar Comisarías de Familia. 
A su vez, esta información fue confirmada por las autoridades entrevistadas. Uno de los participantes adjudica el consumo del alcohol a la cultura, diciendo que "hace parte de la cultura ser bebedor aquí en california", explicando que existe entre los mineros la costumbre de ir a tomar al rancho después de galafardear ${ }^{10}$ (Participante 1, comunicación personal, 7 de febrero de 2018).

Por otro lado, una de las autoridades incluso manifestó que la minería a gran escala aumenta los índices de violencia intrafamiliar, deserción escolar y consumo de alcohol a temprana edad, especialmente entre los adolescentes (Participante 2, comunicación personal, 3 de noviembre de 2018).

En un proceso de observación del contexto de California durante las ferias y fiestas de San Antonio de Padua, santo patrono de los mineros en Santurbán, se pudo observar menores y mayores de edad celebrando por igual en los ranchos (cantinas).

Entonces, el consumo de alcohol y la cultura minera están íntimamente relacionados. Podría decirse que el canon de masculinidad que se da alrededor del alcohol fomenta la violencia intrafamiliar y que es necesario un proceso de responsabilidad social empresarial para que desde las empresas formalmente constituidas en la zona se busque redefinir lo que significa ser minero para aminorar la construcción de valores sociales negativos.

Esta situación debe ser asumida también por el Estado, que debe dar cuenta de la afectación que generan los procesos de delimitación del páramo de Santurbán en la vida de las mujeres. Como se explicó al inicio de este acápite, las denuncias se incrementaron a partir del año 2011 en ambos municipios. En ese mismo año se generó un conflicto con graves secuelas socioambientales y económicas en Vetas y California. Según Basto:

10 El galafardeo es la entrada a los socavones pertenecientes a empresas multinacionales para la extracción de oro de forma clandestina. De acuerdo con lo expresado por los participantes, dicha actividad puede generar altos dividendos y se realiza particularmente de noche. Existen choques entre los galafardos y las multinacionales, ya que esta actividad es considerada ilegal y al mismo tiempo es el medio de subsistencia de muchas familias en Santurbán (Vanguardia Liberal, 2014). 
[...] entre los años 2012 y 2014 las multinacionales que se encontraban en el páramo entraron en stand by por orden del gobierno; esto ocasionó un receso en los modos de subsistencia de las comunidades que históricamente han vivido de la explotación de oro, lo que afectó sus economías y estilos de vida. (Basto, 2017, p. 10)

Al preguntarles a los funcionarios y funcionarias entrevistados sobre la afectación del conflicto socioambiental en la vida de las mujeres, no hubo una respuesta clara, ya que algunos no estaban ejerciendo en el cargo en ese periodo y otros comentaban que las cifras de violencias de género se han mantenido estables. Sin embargo, una funcionaria comentó que en Vetas sí hubo más casos de solicitudes de conciliación por alimentos e inasistencias alimentarias en esta época (Participante No. 3, comunicación personal, 5 de Mayo de 2018).

Por otro lado, en 2014 el comisario de familia de California expresó que el aumento del galafardeo o las prácticas independientes de la minería o "minería ilegal", en el marco de la delimitación del páramo del Santurbán y la declaratoria de parque natural en 2013, han generado dinámicas del consumo de alcohol y de violencia intrafamiliar, por lo que el municipio estaba en alerta para esta época. "Esto está empezando a tomar auge generando violencia intrafamiliar entre los mineros que no saben manejar el dinero y se emborrachan cuando en un solo día les va muy bien" (El Tiempo.com. 2014).

Entonces, se encontraron indicios de la relación entre la minería como práctica económica, el alto consumo de alcohol y la generación de masculinidades violentas que propician la violencia de pareja contra las mujeres.

\section{Las validaciones de la violencia de género en las relaciones de pareja}

Las validaciones detrás de la violencia de pareja son numerosas, pero sobresalen los celos y la intolerancia tanto en Vetas como en California. Los celos tienen que ver con la necesidad de controlar el cuerpo y la vida de la pareja. Por ejemplo, en uno de los casos el agresor le pide a la víctima que no "descuide a la niña" y que se "comporte bien". En 
otras situaciones, el control se observa en la posesión sobre los bienes de la víctima; por ejemplo, en casos donde el agresor ingresa a la casa de la víctima, la suele denominar "nuestra casa", "la casa de nosotros" así ya no convivan (Comisaría de Familia de Vetas y California, 2018).

Los celos llevan a muchos agresores a impedirle a sus parejas salir de la casa o hablar con otros hombres, especialmente cuando ellos están trabajando. En un caso, por ejemplo, el agresor abusa sexualmente de su pareja constantemente porque ella sale de la casa (Comisaría de Familia de Vetas y California, 2018).

Algunas mujeres justifican las agresiones que han sufrido con $\mathrm{CO}^{-}$ mentarios alrededor de su naturaleza de celosas; ejemplo de ello es esta mujer que dice: "Es que yo antes creía mucho en chismes y ahora él no me cree nada cuando estamos solos me dice que no le hable" (Comisaría de Familia de Vetas y California, 2018).

De otra parte, la intolerancia tiene que ver con el escalamiento de la violencia de pareja por motivos fútiles, como encontrar la puerta cerrada de la casa, perder dinero, hablar con amigos o familia o "echarle cantaleta". Sin embargo, si se indagan a profundidad estos casos con motivos aparentemente superficiales, se encuentra entonces un sustrato profundamente patriarcal.

Además de justificar la violencia con los motivos mencionados, los agresores suelen invisibilizar ciertas situaciones de violencia con expresiones como "eso fue de parte y parte" o "yo la grite pero no la amenacé", "yo le di correa pero no pata (sic)" (Comisaría de Familia de Vetas y California, 2018).

Existe un patrón alrededor de las maternidades y es la manipulación de parte del agresor a la víctima en términos de que si sale de la casa sola o interactúa con otros hombres entonces merece ser agredida, ya que se ha convertido en una mala madre, por lo que es usual expresiones como "es que ella deja abandonados a los niños". Ello también aplica cuando las mujeres terminan las relaciones afectivas. Esto ocurrió en 11 de 24 casos en California y en 8 de 35 casos en Vetas (Comisaría de Familia de Vetas y California, 2018). 


\section{Conclusiones}

1. No existen estadísticas unificadas y oficiales de la violencia de género en el país y menos de la violencia de pareja, ni siquiera cuando se toma una misma variable como es el denuncio de un delito. La Fiscalía General de la Nación no realiza sus estadísticas en coordinación con las comisarías de familia, a pesar de que todos los casos recibidos por las comisarías de familia deben ser reportados a la Fiscalía. Tampoco existe de parte de ninguna de las dos entidades una metodología seria para la construcción de variables que permitan dilucidar los factores que, desde una perspectiva de género, intersectan con la violencia de género. Lo anterior afecta la posibilidad de realizar investigaciones y políticas públicas efectivas sobre la violencia de género, ya que no hay un diagnóstico serio de la realidad social.

Al mismo tiempo, los intentos de construcción de panoramas criminológicos de la violencia de género se han restringido a las capitales de departamentos, dejando de lado las dinámicas sociales de las regiones más apartadas. Entonces, las políticas públicas no solo carecen de un diagnóstico serio de la realidad social, sino que son centralistas.

2. Uno de los factores que se convierte en sustrato de la violencia de pareja en el municipio tiene que ver con la fuerte división sexual del trabajo en los municipios estudiados. Lo anterior genera dependencia económica, ya que la violencia económica, a pesar de estar invisibilizada, es la más recurrente junto con la violencia física y la psicológica en las mujeres de los municipios estudiados. Esto se suma a la baja escolaridad y a que estas mujeres son amas de casa o ejercen profesiones poco remuneradas. La dependencia mencionada se exacerba en contextos de crisis y de desempleo; inclusive, el número de conciliaciones de alimentos aumentó en los últimos años de acuerdo con lo establecido por los comisarios de familia de Vetas y California, quienes relacionan este fenómeno a la delimitación del páramo de Santurbán a partir de 2011 y el aumento del galafardeo.

3. La intersección de la minería, el alcohol y la violencia de pareja es clara, ya que dentro de la cultura minera del municipio está el consumo de sustancias alcohólicas, elemento fundamental en los casos de violencia de pareja. Los participantes incluso aseveraron en en- 
trevista que la minería informal propicia el aumento de situaciones de violencia de pareja y el consumo de alcohol. En este contexto, la familia es la instancia de control social de las mujeres, en la cual mediante el chisme, el rumor, y la vigilancia se determina qué mujeres salen solas de su casa, socializan con hombres y rompen relaciones afectivas para, de esta forma, sancionarlas por medio de la violencia de pareja.

\section{Referencias}

Alcaldía de Vetas. (2012). Plan de desarrollo 2012-2015. Recuperado de http:// cdim.esap.edu.co/bancomedios/Documentos\%20PDF/vetassantanderpd20122015.pdf

Alcenar-Rodriguez, C. (2012). Violencia de género en la pareja: una revisión teórica. Revista PsiCo, (43), 116-126.

Álvarez, A. (2017). Memoria de las prácticas mineras en el municipio de California para fortalecer la educación ambiental (Tesis de Maestría), Universidad Santo Tomás, Bucaramanga, Colombia.

Basto, S. (2017). El conflicto socioambiental del páramo Santurbán. Un análisis bioético con enfoque de ecología política. Revista Colombiana de Bioética, 12(1), 8-24.

BBC. (2016). América Latina: Alianza Siniestra entre la Minería Ilegal y la Trata de Mujeres. Recuperado de https://www.bbc.com/mundo/noticias/2016/04/160406_ america_latina_alianza_siniestra_mineria_ilegal_trata_mujeres_prostitucion_sexual_bm

Bermúdez, R., Rodríguez Maldonado, T., \& Roa Avendaño, T. (2012). Mujer y minería. Ámbitos de análisis e Impactos de la minería en la vida de las mujeres. CENSAT AGUAVIVA. P 1-23. Recuperado de http://desterresminees. pasc.ca/wp-content/uploads/2015/11/Bermudez-Rico-et-al-2011-Mujer_y_ Mineria.pdf

Bourdieu, P. (2000). La dominación Masculina. Barcelona: Editorial Anagrama.

Buitrago, E. (2012). Entre el agua y el oro: tensiones y reconfiguraciones territoriales en el municipio de Vetas, Santander (tesis de grado). Universidad Nacional Bogotá; Colombia.

Chaparro, E. (2005). La mujer en la pequeña minería de América Latina: el caso de Bolivia (Volumen 87). Bolivia: Editorial United Nations Publications. 
CIDH. (2001). Caso María da Penha Maia Fernández Vs Brasil. Recuperado de https://www.cidh.oas.org/annualrep/2000sp/CapituloIII/Fondo/Brasil12.051. htm

CIDH. (2007). Acceso a la justicia para las mujeres víctimas de violencia en las Américas, OEA/Ser.L/V/II. Doc. 68, 20 enero 2007.

Colombia. Ministerio de Minas y Energía. (2016). Política Minera de Colombia. Bases para la Minería del futuro. Bogotá: El Ministerio.

Contreras. (2014). La familia y los amigos: los vigilantes de las normativas de género y sexualidad para una mujer joven del Norte Minero de Chile. En Complejidad y desafíos de la transformación social De la ciencia a la agencia: Actas Científicas del Centro de Estudios Sociales y Región, (pp. 437-451). Guadalajara: Universidad de Guadalajara.

Cook \& Cusack. (2011). Estereotipos de Género, perspectivas legales trasnacionales. Bogotá: Editorial siglo del hombre.

El Espectador. (2018). Impunidad: El capítulo colombiano de los ambientalistas asesinados. Recuperado de https://colombia2020.elespectador.com/pais/ impunidad-el-capitulo-colombiano-de-los-ambientalistas-asesinados.

El Tiempo.com. (2014). Aumenta la Minería Ilegal en Santurbán. Recuperado de https://www.eltiempo.com/archivo/documento/CMS-14055156.

Facio, A. E Fries, L. (2005). Feminismo Género y Patriarcado. En Academia Revista sobre la enseñanza del derecho de Buenos Aires (pp. 259-294). Ciudad: Buenos Aires. Universidad de Buenos Aires

Fairclough, N. E Wodak, R. (1997). Critical Discourse Analysis. Discourse a s a social interaction. Discourse studies: A multidisciplinary introduction. Thousand Oaks, CA: Sage.

Federici. (2013). Revolución en punto cero. Trabajo doméstico, reproducción y luchas feministas. Madrid: Editorial Traficantes de sueños.

Galtung, J (1989). La Violencia Cultural. Bizkaia: Vizcaya Centro de Investigación por la paz.

Gartor, Miriam. (2014) “El feminismo reactiva la lucha contra el extractivismo en América Latina". En La Marea. Revista virtual. Recuperado de http://www. lamarea.com/2014/02/17/ecuador-extractivismo-mujeres/

Güiza, L. (2013). La pequeña minería en Colombia: una actividad no tan pequeña. Dyna, (181), 109-117.

CNMH. (2014). Silenciar la democracia: las masacres de Remedios y Segovia, 1982-1997. Ciudad: Bogotá editorial CNMH. (2016ª). Petróleo, coca, despojo territorial y organización social en el putumayo. Ciudad: Bogotá editorial CNMH. 
(2016b) La maldita tierra, guerrilla, paramilitares, mineras y conflicto armado en el departamento del Cesar. Bogotá: editorial CNMH.

INMLCF. (2015). Forensis Masatugó. Recuperado de http://www.medicinalegal.gov.co/documents/20143/56654/5+Masatugo+relaciones+pare ja+2009-2014.pdf

Maqueda, M. (2006). La Violencia de Género: Entre el concepto Jurídico y la Realidad Social. Revista Electrónica de Ciencia y Tecnología, 8(2), 1-13.

Méndez, R., Rojas, H., E Montero, L. (2016). Tensiones identitarias en las vivencias erótico-afectivas: escenario cotidiano de las relaciones de género en contextos extractivos de oro y petróleo. Revista Tábula Rasa, (26), 331-351.

OEA. (1969). Convención Americana de Derechos Humanos. Recuperado de https:// www.oas.org/dil/esp/tratados_b-32_convencion_americana_sobre_derechos_humanos.htm.

OEA. (1994). Convención Interamericana para prevenir, sancionar y erradicar la violencia contra la mujer. "Convención Belem do Pará". Recuperado de http://www.oas.org/juridico/spanish/tratados/a-61.html

ONU. (1993). Declaración para la eliminación de la violencia contra la mujer. Belem do Pará: ONU.

Parra, J. (2006). Familia, poder y esmeraldas. Relaciones de género y estructura económica minera en el occidente de Boyacá, Colombia. Revista Colombiana de Antropología. (42), 15-54.

Pineda, J. E Otero, L. (2004). Género, Violencia Intrafamiliar e Intervención Pública en Colombia. Revista de Estudios Sociales, (17), 19-31.

Red Nacional de Mujeres. (2015). Mujeres, Conflictos Socioambientales y Resolución 1325 de las Naciones Unidas. Bogotá: Red Nacional de Mujeres.

Scott, J. (1996). El género: una categoría útil para el análisis histórico. En: Lamas Marta Compiladora. El género: la construcción cultural de la diferencia sexual. PUEG, México.

Vanguardia Liberal. (2018). Mas del 80\% de la población de Vetas se encuentra sin empleo. Recuperado de http://www.vanguardia.com/economia/local/437409mas-del-80-de-la-poblacion-de-vetas-santander-se-encuentra-sin-empleo. 